\title{
Perfil epidemiológico da diarreia em crianças de 1 a 4 anos no estado de Alagoas
}

\author{
Epidemiological profile of diarrhea in children 1 to 4 years old in the state of Alagoas
}

\author{
Caroline Magna de Oliveira Costa ${ }^{1}$ \\ Orcid: https://orcid.org/0000-0003-4096-4093
}

Thaynara Maria Pontes Bulhões ${ }^{3}$

Orcid: https://orcid.org/0000-0003-2398-8173

Beatriz Rodrigues Gusmão 5

Orcid: https://orcid.org/0000-0003-2892-1234

Roberta Zaninelli do Nascimento ${ }^{7}$

Orcid: https://orcid.org/0000-0003-4256-8633
Diane Fernandes dos Santos ${ }^{2}$

Orcid: https://orcid.org/0000-0002-5472-4122

Jayane Omena de Oliveira ${ }^{4}$

Orcid: https://orcid.org/0000-0003-2830-9379

Juliana Barbosa Valdevino de Oliveira ${ }^{6}$

Orcid: https://orcid.org/0000-0002-7446-7266

Christefany Régia Braz Costa ${ }^{8}$

Orcid: https://orcid.org/0000-0001-6124-8243

\section{Resumo}

Introdução: A diarreia se caracteriza por evacuações recorrentes, podendo ser soltas ou líquidas, e é a segunda causa de morte em menores de cinco anos, além de ser responsável por um terço das internações. Dentre os nove estados da região Nordeste, Alagoas, é o segundo estado com o menor número de municípios que possuem algum tipo de plano de saneamento, o que impacta negativamente na saúde da população. Objetivo: Traçar o perfil epidemiológico da diarreia entre crianças de 1 a 4 anos no estado de Alagoas no período de janeiro de 2014 a dezembro de 2018. Métodos: trata-se de estudo retrospectivo, descritivo, de abordagem quantitativa, realizado no período de outubro de 2019 a janeiro de 2020 por meio do Sistema de Informações Hospitalares do SUS (SIH/SUS), disponível no site do DATASUS. Resultados: no período de janeiro de 2014 a dezembro de 2018, o número de internações hospitalares por diarreia e gastroenterites de origem infecciosa presumível no Estado de Alagoas, foi relativamente equivalente entre os sexos, com predominância de crianças pardas, Arapiraca Foi o município com maiores notificações e, no geral houve quatro óbitos. Conclusão: Dessa forma, os resultados sinalizam para a necessidade de maior aprofundamento no estudo das variáveis que se relacionam as internações hospitalares por diarreia e gastroenterites, para a formulação de intervenções em saúde voltadas à prevenção desse agravo.

Palavras-chave: diarreia; diarreia infantil; criança; epidemiologia.

\footnotetext{
Abstract

Introduction: Diarrheais characterized by recurrent loose or liquid bowel movements. It is the second main cause of deathin children under 5 years old, being also responsible for one third of their hospitalizations. Of the nine states in the Northeast Region, Alagoas has the second

${ }^{1}$ Universidade Federal de Alagoas. Maceió, Alagoas, Brasil. E-mail: carolmagnacosta@gmail.com

${ }^{2}$ Universidade Federal de Alagoas. Maceió, Alagoas, Brasil. E-mail: dianefernandes@outlook.com.br

${ }^{3}$ Universidade Federal de Alagoas. Maceió, Alagoas, Brasil. E-mail: thaynarabulhões@hotmail.com

${ }^{4}$ Universidade Federal de Alagoas. Maceió, Alagoas, Brasil. E-mail: jayaneomena@gmail.com

${ }^{5}$ Universidade Federal de Alagoas. Maceió, Alagoas, Brasil. E-mail: bearodriguesg@gmail.com

${ }^{6}$ Universidade Federal de Alagoas. Maceió, Alagoas, Brasil. E-mail: julianabvo.enf@gmail.com

${ }^{7}$ Universidade Federal de Alagoas. Maceió, Alagoas, Brasil. E-mail: roberta.zaninelli@esenfar.ufal.br

8 Universidade Federal de Alagoas. Maceió, Alagoas, Brasil. E-mail: christefany.costa@usp.br
} 
fewest municipalities with any type of sanitation planning - which has a negative impact on the population's health. Objective: To outline the epidemiological profile of diarrhea in children 1 to 4 years oldin Alagoas between January 2014 and December 2018. Methods: This is a retrospective, descriptive study with a quantitative approach, conducted from October 2019 to January 2020 with data from the Hospital Information System of SUS (SIH/SUS), available at the DATASUS website. Results: Between January 2014 and December 2018, the number of hospitalizations due to presumable infectious diarrhea and gastroenteritis in Alagoas was relatively equivalent between the sexes, with a predominance of multiracial children. Arapiraca was the municipality with the most reports. Altogether, there were four cases of death. Conclusion: The results point to the need for a more in-depth study of the variables related to hospitalizations due to diarrhea and gastroenteritis, and thus develop health interventions to prevent this disease.

Keywords: diarrhea; diarrhea, infantile; child; epidemiology.

\section{Introdução}

\begin{abstract}
A gastroenterite é um termo associado a patologias no trato gastrointestinal que podem ter origem infecciosa parasitária, bacteriana ou viral, cuja diarreia é a sua principal manifestação. A diarreia caracteriza-se por evacuações recorrentes, que se apresentam nas formas solta ou líquida ${ }^{1}$.
\end{abstract}

Em uma perspectiva global, segundo a Organização Mundial da Saúde (OMS) e o Fundo das Nações Unidas para a Infância (UNICEF),ocorrem cerca de 2 bilhões de casos de doenças diarreicas em todo o mundo, sendo 1,9 milhão de óbitos em crianças menores de 5 anos nos países em desenvolvimento. A diarreia aguda configura-se como a segunda maior causa de morte nessa faixa etária e é responsável por um terço das internações. Esse agravo compromete a saúde e o desenvolvimento infantil, uma vez que desencadeia situações de desnutrição e desidratação ${ }^{2,3}$.

A ocorrência da diarreia na infância está associada a fatores biológicos, sociais, ambientais, sanitários, econômicos e culturais $^{4}$. Nesse contexto, o saneamento básico no Brasil ganha destaque, especialmente no Nordeste, por sua precariedade. Essa condição comumente encontrada, associada a uma falta de acesso a informação e orientação, repercute na saúde da população com baixas condições econômicas, tornando-as mais suscetíveis ao acometimento da doença ${ }^{5}$.
Ademais, de acordo com dados do Instituto Brasileiro de Geografia e Estatística (IBGE), dentre os nove estados da região Nordeste, Alagoas, que possui 102 municípios, é o segundo estado com o menor número de cidades que possuem algum tipo de plano de saneamento ${ }^{6}$. Desse modo, há um impacto negativo na qualidade de vida da população alagoana, já que segundo a OMS, o saneamento básico é uma das principais formas de prevenção de agravos, incluindo as diarreias infecciosas ${ }^{5}$.

Tendo em vista as implicações trazidas pela diarreia e os fatores que favorecem o seu acometimento, é importante investigar variáveis que influenciam sua incidência, a fim de subsidiar a elaboração de estratégias para prevenção e promoção da saúde na infância, sobretudo nas doenças prevalentes, como a diarreia. Sendo assim, este estudo tem como objetivo traçar o perfil epidemiológico da diarreia entre crianças de 1 a 4 anos no estado de Alagoas no período de janeiro de 2014 a dezembro de 2018.

\section{Materiais e Métodos}

\section{Amostra e tipo de estudo}

Trata-se de um estudo retrospectivo, descritivo, de abordagem quantitativa, com 4379 casos notificados de diarreia em criança de 1 a 4 anos no estado de Alagoas, entre o período de janeiro de 2014 a dezembro de 2018 . 


\section{Delineamento da pesquisa}

A coleta de dados foi realizada por meio do Sistema de Informações Hospitalares do SUS (SIH/SUS), disponível no site do Departamento de Informática do Sistema Único de Saúde $^{7}$ (DATASUS). Os dados foram coletados de outubro de 2019 até janeiro de 2020.

\section{Critérios de Inclusão e Exclusão}

Avaliou-se o número de internações hospitalares por diarreia e gastroenterite de origem infecciosa presumível em crianças de 1 a 4 anos no Estado de Alagoas, referente aos últimos cinco anos, período de janeiro de 2014 a dezembro de 2018. As variáveis investigadas foram: faixa etária (1-4 anos), sexo (masculino e feminino), cor/raça (branca, preta, parda e amarela), ano de notificação (2014-2018), número de óbitos e taxa de mortalidade, a qual é calculada a partir da proporção do número de óbitos no período, pela população no meio do período, multiplicado por uma constante ${ }^{8}$. Optou-se por investigar essa faixa por ser uma variável fixa no DATASUS e ser o público mais acometido por essa patologia. Ademais, o estado apresenta 102 municípios, contudo, este estudo utilizou informações de 33, uma vez que eram os únicos disponíveis no DATASUS. A saber: Água Branca, Anadia, Arapiraca, Atalaia, Batalha, Boca da Mata, Cajueiro, Campo Alegre, Capela, Coruripe, Girau do Ponciano, Igaci, Limoeiro de Anadia, Joaquim Gomes, Junqueiro, Maceió, Matriz de Camaragibe, Murici, Olho d'Água das Flores, Palmeira dos Índios, Pão de Açúcar, Penedo, Pilar, Piranhas, Porto Calvo, Quebrangulo, Rio Largo, Santana do
Ipanema, São José da Laje, São Luis do Quitunde, São Miguel dos Campos, Teotônio Vilela, União dos Palmares.

\section{Procedimentos}

Nesse contexto, para a obtenção dos dados, realizou-se o cruzamento das seguintes variáveis: número de internações por diarreia e gastroenterite de origem infecciosa presumível, faixa etária de 1-4 anos, municípios do Estado de Alagoas, sexo e cor/raça dos menores acometidos. Posteriormente, os dados foram tabulados e analisados através do programa Microsoft Excel. Por se tratar de dados secundários de domínio público, não houve necessidade de encaminhar o estudo para o Comitê de Ética em Pesquisa (CEP). As Normas e Diretrizes Regulamentadoras da Pesquisa Envolvendo Seres Humanos Resolução CNS 466/12, foram respeitadas.

\section{Resultados}

Observou-se que no período de 2014 a 2018, no Estado de Alagoas, o número de internações hospitalares por diarreia e gastroenterites de origem infecciosa presumível entre crianças de 1 a 4 anos foi relativamente equivalente entre os sexos, com um total de $\mathrm{n}=2206$ internações $(50,4 \%)$ para o sexo feminino e $n=2173$ internações $(49,6 \%)$ para o sexo masculino. Além disso, entre as crianças internadas, predominou-se a cor/raça parda, com um total de $n=3429(78,3 \%)$, destacando que $\mathrm{n}=765(17,5 \%)$ dos casos notificados não informaram essa variável, conforme mostra a tabela 1.Das 4379 notificações entre 2014 e 2018, houve 4 óbitos e não há registros de óbitos nos anos de 2015 e 2017, apresentando taxa de mortalidade de 0,94. 
Tabela 1 -Número de Internações por Diarreia e Gastroenterites de origem Infecciosa presumível no Estado de Alagoas entre Crianças de 1-4 anos, de acordo com dados sociodemográficos. Alagoas, Brasil, 2014-2018. $(\mathrm{n}=4379)$

\begin{tabular}{|c|c|c|c|c|c|c|c|c|c|}
\hline \multicolumn{10}{|c|}{ Ano de notificação } \\
\hline & \multicolumn{2}{|c|}{2014} & \multicolumn{2}{|c|}{2015} & \multicolumn{2}{|c|}{2016} & \multicolumn{2}{|c|}{2017} & 2018 \\
\hline Variáveis & $\mathrm{n}$ & $\%$ & $\mathrm{n}$ & $\%$ & $\mathrm{n}$ & $\%$ & $\mathrm{n}$ & $\%$ & $\mathrm{n}$ \\
\hline
\end{tabular}

\begin{tabular}{|c|c|c|c|c|c|c|c|c|c|c|c|c|}
\hline \multicolumn{13}{|l|}{ Sexo } \\
\hline Masculino & 554 & 50,2 & 368 & 49,1 & 432 & 50,6 & 449 & 47,3 & 370 & 51,2 & 2173 & 49,6 \\
\hline Feminino & 550 & 49,8 & 381 & 50,9 & 422 & 49,4 & 501 & 52,7 & 352 & 48,8 & 2206 & 50,4 \\
\hline Total & 1104 & 100 & 749 & 100 & 854 & 100 & 950 & 100 & 722 & 100 & 4379 & 100 \\
\hline \multicolumn{13}{|l|}{ Cor/Raça } \\
\hline Branca & 36 & 3,3 & 21 & 2,80 & 31 & 3,6 & 35 & 3,7 & 24 & 3,3 & 147 & 3,3 \\
\hline Preta & 2 & 0,2 & 2 & 0,3 & 2 & 0,2 & 14 & 1,5 & 1 & 0,2 & 21 & 0,5 \\
\hline Parda & 781 & 70,7 & 503 & 67,1 & 655 & 76,7 & 838 & 88,2 & 652 & 90,3 & 3429 & 78,3 \\
\hline Amarela & 5 & 0,4 & 5 & 0,7 & 4 & 0,5 & 3 & 0,3 & - & - & 17 & 0,4 \\
\hline $\begin{array}{c}\text { Sem } \\
\text { informação }\end{array}$ & 280 & 25,4 & 218 & 29,1 & 162 & 19 & 60 & 6,3 & 45 & 6,2 & 765 & 17,5 \\
\hline Total & 1104 & 100 & 749 & 100 & 854 & 100 & 950 & 100 & 722 & 100 & 4379 & 100 \\
\hline
\end{tabular}

Fonte: Elaborada pelos autores com base no DATASUS, 2020.

Verificou-se também que, dentre os 33 municípios do Estado de Alagoas que notificaram o agravo no período de janeiro de 2014 a dezembro de 2018, Arapiraca foi o município que obteve o maior destaque, apresentando $\mathrm{n}=2275$ internações $(52,0 \%)$. Em contrapartida, 10 outros municípios obtiveram um número igual ou menor que 10 de internações registradas (tabela 2).

Tabela 2 -Número de Internações por Diarreia e Gastroenterites de origem Infecciosa presumível no Estado de Alagoas entre Crianças de $1-4$ anos, de acordo com os Municípios. Alagoas, Brasil, 2014-2018. (n=4379)

\begin{tabular}{|c|c|c|c|c|c|c|c|c|c|c|c|c|}
\hline \multicolumn{13}{|c|}{ Ano de notificação } \\
\hline & \multicolumn{2}{|c|}{2014} & \multicolumn{2}{|c|}{2015} & \multicolumn{2}{|c|}{2016} & \multicolumn{2}{|c|}{2017} & \multicolumn{2}{|c|}{2018} & \multicolumn{2}{|c|}{ Total } \\
\hline $\begin{array}{c}\text { Variáveis } \\
\text { Municípios }\end{array}$ & $\mathrm{n}$ & $\%$ & $\mathrm{n}$ & $\%$ & $\mathrm{n}$ & $\%$ & $\mathrm{n}$ & $\%$ & $\mathrm{n}$ & $\%$ & $\mathrm{n}$ & $\%$ \\
\hline $\begin{array}{l}\text { Água } \\
\text { Branca }\end{array}$ & - & - & - & - & - & - & 3 & 0,3 & 2 & 0,3 & 5 & 0,1 \\
\hline Anadia & 1 & 0,1 & - & - & - & - & - & - & - & - & 1 & 00 \\
\hline Arapiraca & 521 & 47,2 & 310 & 41,4 & 459 & 53,8 & 579 & 61 & 406 & 56,2 & 2275 & 52 \\
\hline Atalaia & 40 & 3,6 & 17 & 2,3 & 7 & 0,8 & 3 & 0,3 & - & - & 67 & 1,5 \\
\hline Batalha & 69 & 6,2 & 33 & 4,4 & 15 & 1,8 & 3 & 0,3 & 2 & 0,3 & 122 & 2,8 \\
\hline $\begin{array}{c}\text { Boca da } \\
\text { Mata }\end{array}$ & 10 & 1,0 & 10 & 1,3 & 1 & 0,1 & - & - & 1 & 0,1 & 22 & 0,5 \\
\hline Cajueiro & 4 & 0,4 & 2 & 0,3 & 1 & 0,1 & - & - & - & - & 7 & 0,2 \\
\hline $\begin{array}{l}\text { Campo } \\
\text { Alegre }\end{array}$ & 1 & 0,1 & 7 & 0,9 & 8 & 0,9 & 5 & 0,5 & 2 & 0,3 & 23 & 0,5 \\
\hline Capela & 6 & 0,5 & 9 & 1,2 & 3 & 0,4 & 2 & 0,2 & 5 & 0,7 & 25 & 0,6 \\
\hline Coruripe & 1 & 0,1 & 2 & 0,3 & 6 & 0,7 & 2 & 0,2 & 6 & 0,9 & 17 & 0,4 \\
\hline $\begin{array}{l}\text { Girau do } \\
\text { Ponciano }\end{array}$ & 3 & 0,3 & - & - & - & - & - & - & - & - & 3 & 0,1 \\
\hline Igaci & 7 & 0,6 & - & - & - & - & - & - & - & - & 7 & 0,2 \\
\hline $\begin{array}{c}\text { Limoeiro de } \\
\text { Anadia }\end{array}$ & 4 & 0,4 & 1 & 0,1 & - & - & - & - & - & - & 5 & 0,1 \\
\hline $\begin{array}{l}\text { Joaquim } \\
\text { Gomes }\end{array}$ & 6 & 0,5 & - & - & 2 & 0,2 & 3 & 0,3 & 1 & 0,1 & 12 & 0,3 \\
\hline Junqueiro & 1 & 0,1 & - & - & - & - & - & - & - & - & 1 & 00 \\
\hline
\end{tabular}




\begin{tabular}{|c|c|c|c|c|c|c|c|c|c|c|c|c|}
\hline Maceió & - & - & 3 & 0,4 & 8 & 0,9 & 16 & 1,7 & 17 & 2,4 & 44 & 1,0 \\
\hline Matriz de & 1 & 0,1 & 3 & 0,4 & 1 & 0,1 & 3 & 0,3 & 2 & 0,3 & 10 & 0,2 \\
\hline \multicolumn{13}{|l|}{ Camaragibe } \\
\hline Murici & 18 & 1,6 & 14 & 1,9 & 10 & 1,2 & - & - & - & - & 42 & 1,0 \\
\hline Olho & 1 & 0,1 & 3 & 0,4 & 5 & 0,6 & - & - & - & - & 9 & 0,2 \\
\hline \multicolumn{13}{|l|}{$\begin{array}{l}\text { d'Água das } \\
\text { Flores }\end{array}$} \\
\hline $\begin{array}{l}\text { Palmeira dos } \\
\text { Índios }\end{array}$ & 62 & 5,6 & 35 & 4,7 & 72 & 8,4 & 65 & 6,9 & 65 & 9,0 & 299 & 6,8 \\
\hline $\begin{array}{l}\text { Pão de } \\
\text { Acúcar }\end{array}$ & 25 & 2,2 & 19 & 2,5 & 17 & 2,0 & 9 & 1,0 & 7 & 1,0 & 77 & 1,8 \\
\hline Penedo & 1 & 0,1 & - & - & - & - & - & - & 11 & 1,5 & 12 & 3 \\
\hline Pilar & 24 & 2,2 & 26 & 3,5 & - & - & 57 & 6,0 & 48 & 6,6 & 155 & 5 \\
\hline Piranhas & 41 & 3,7 & 58 & 7,7 & 47 & 5,5 & 48 & 5,0 & 68 & 9,4 & 262 & 0 \\
\hline Porto Calvo & - & - & - & - & - & - & 19 & 2,0 & 7 & 1,0 & 26 & 6 \\
\hline Quebrangulo & 12 & 1,1 & 7 & 0,9 & 13 & 1,5 & 25 & 2,6 & 9 & 1,3 & 66 & 5 \\
\hline Rio Largo & 2 & 0,2 & 3 & 0,4 & 1 & 0,1 & 2 & 0,2 & 2 & 0,3 & 10 & . \\
\hline $\begin{array}{l}\text { Santana do } \\
\text { Ipanema }\end{array}$ & 29 & 2,6 & 18 & 2,4 & 10 & 1,2 & 24 & 2,5 & 11 & 1,5 & 92 & \\
\hline $\begin{array}{l}\text { São José da } \\
\text { Laje }\end{array}$ & - & - & 2 & 0,3 & 5 & 0,6 & 5 & 0,5 & 2 & 0,3 & 14 & \\
\hline $\begin{array}{l}\text { São Luis do } \\
\text { Quitunde }\end{array}$ & 23 & 2,1 & 4 & 0,5 & - & - & - & - & 1 & 0,1 & 28 & \\
\hline $\begin{array}{l}\text { São Miguel } \\
\text { dos Campos }\end{array}$ & 151 & 13,7 & 112 & 15 & 105 & 12,3 & 31 & 3,3 & 22 & 3,0 & 421 & \\
\hline $\begin{array}{c}\text { Teotônio } \\
\text { Vilela }\end{array}$ & 13 & 1,2 & 17 & 2,3 & 17 & 2,0 & 31 & 3,3 & 22 & 3,0 & 100 & \\
\hline União dos & 27 & 2,4 & 34 & 4,5 & 41 & 4,8 & 15 & 1,6 & 3 & 0,4 & 120 & \\
\hline \multicolumn{12}{|l|}{ Palmares } & \\
\hline Total & 1104 & 100 & 749 & 100 & 854 & 100 & 950 & 100 & 722 & 100 & 4379 & \\
\hline
\end{tabular}

\section{Discussão}

O presente estudo identificou que o número de internações hospitalares por diarreias de origem infecciosa entre os menores de cinco anos ainda atinge proporções relevantes, corroborando, assim, com outros estudos realizados em todo o território brasileiro ${ }^{5,9,10}$. Desse modo, vale salientar que o maior acometimento dessa faixa etária (de 1 a 4 anos de idade) está relacionado coma imaturidade do sistema imunológico e aos determinantes sociais de saúde ao qual a criança está inserida, pois estes impactam diretamente no acometimento desta doença ${ }^{11}$.

Ademais, foi observado com esta pesquisa que o número de internações hospitalares por diarreia e gastroenterites de origem infecciosa presumível entre crianças de 1 a 4 anos no estado de Alagoas foi relativamente equivalente entre os sexos.
Um estudo realizado por Rocha na Bahia ${ }^{12}$, região nordeste do Brasil, avaliou o perfil epidemiológico e etiológico de 499 crianças com diarreia aguda em unidades de emergência pediátrica, e demonstrou um resultado de $52,5 \%$ para o sexo feminino e 47,5\% para o masculino. Em contrapartida, uma pesquisa realizada em São Paulo, que buscou conhecer as características epidemiológicas desta patologia no município de Avaré, gerou um percentual de $54,0 \%$ para o sexo masculino e $46,0 \%$ para o feminino, entre os 408 investigados $^{13}$. Interessante destacar que, apesar da distinção entre os estudos realizados em outras regiões, a diferença no sexo não é algo predominante nos estudos, o que pode sinalizar que essa variável não tem tanta implicação na condição de ser ou não mais susceptível a diarreia.

Quanto à classificação étnico-racial, notou-se que, entre as crianças internadas, 
houve predomínio da cor/raça parda, ao passo que em 17,5\% não foram informados. Entretanto, quando comparado com a literatura disponível, percebe-se a falta de estudos que abordam a relação dessa variável com a internação por diarreia, necessitando-se, assim, de estudos que abordem essa questão, sobretudo quando se trata da população brasileira e sua heterogeneidade frente a cor/raça e situação de vulnerabilidade social.

No que tange aos municípios, Arapiraca apresentou 2.275 do total de 4.379 internações por diarreia e gastroenterites, representando o município com o maior número de notificações do estado. $\mathrm{O}$ rápido crescimento do munícipio nos últimos anos implicou em um não planejamento do desenvolvimento urbano e dos recursos hídricos locais, resultando em agravos relacionados à saúde. Atualmente, estima-se que o município possua uma população de 231.747 habitantes ${ }^{14}$. Os resultados apontam para necessidade de um desenvolvimento sustentável, em que o meio ambiente, a sociedade e a economia estejam interligadas, já que, um mal planejamento hídrico e a distribuição de água de baixa qualidade, podem ser potenciais transmissores para doenças, em especial a diarreia ${ }^{15}$. Vale ressaltar que em 2013, houve um surto de diarreia no estado de Alagoas e, dentre os 25 municípios notificados, Arapiraca apresentou o segundo maior número de óbitos por diarreia ${ }^{16}$.

Somado a isso, o modelo de regionalização em saúde no estado de Alagoas, que é baseado em critérios de densidade demográfica e tecnológica de atenção especializada e hospitalar, define o município de Arapiraca como sede da segunda macrorregião de saúde no estado, abrangendo quatro regiões de saúde e sendo referência para 46 municípios ${ }^{17}$. Dessa maneira, o conjunto de fatores relacionados à quantidade populacional, o desenvolvimento urbano ineficaz e a posição de referência em serviços de saúde no estado, podem justificar o fato desse ser o município mais acometido por diarreia nos últimos cinco anos, quando comparado aos outros 33 municípios notificados.

Ao comparar o índice de internação por diarreia em crianças de 1 a 4 anos nos municípios de Alagoas, observou-se que, dos 102 municípios presentes no estado, apenas 33 notificaram o número de internações decorrente a diarreia, ao passo que 10 apresentaram um número igual ou menor que 10 notificações registradas.Das4379 notificações entre 2014 e 2018, houve 4 óbitos e não há registros de óbitos nos anos de 2015 e 2017. Situação semelhante, no que diz respeito à relação entre casos notificados e óbitos, é observada no estudo de Meneguessi et al. $(2015)^{18}$, realizado no Distrito Federal, onde foi constatado um número baixo de óbitos por diarreia por causa presumível em crianças de 1 a 4 anos. Tal resultado é explicado pelo aumento no acesso aos serviços de saúde, melhoria na qualidade de atendimento à criança, incentivo ao uso de terapia de reidratação oral, dentre outros. Entretanto, questiona-se a real representatividade desses dados, considerando o contexto social e econômico do estado, marcado pela vulnerabilidade social da população.

Nesse contexto, quando este sistema falha, isto é, quando se tem um número insuficiente de notificações, há, por consequência, a não geração de informações, sendo estas extremamente necessárias para orientar a prevenção de doenças, a gestão de serviços, a implantação, o acompanhamento e a avaliação das ações em saúde ${ }^{19}$.Além disso, faz-se necessário, também, que profissionais da área da saúde, inclusive o enfermeiro, sejam capacitados para observar todas as variáveis que tangem essa patologia, para que exerçam práticas de cuidado integral, associado com técnicas intersubjetivas e dialógicas. É imprescindível o rompimento do modelo biomédico e análise de todos os fatores relacionados ao processo saúde-doença ${ }^{20}$. 
enfrentou algumas limitações, principalmente relacionada à incipiência de artigos científicos que abordassem a relação das variáveis sexo, idade e classificação étnico-racial com as internações hospitalares por diarreia e gastroenterites de origem infecciosa presumível, além do registro nulo de algumas variáveis analisadas, possivelmente devido a subnotificação. Desse modo, faz-se necessário mais estudos sobre a temática, a fim de melhorar/qualificar a caracterização do perfil epidemiológico e estimular uma visão crítica por parte dos profissionais da saúde para este agravo.

\section{Conclusão}

O perfil epidemiológico da diarreia entre crianças de 1 a 4 anos no estado de Alagoas, no período de janeiro de 2014 a dezembro de 2018, foi caracterizado pela incidência relativamente equivalente entre os sexos feminino e masculino, com predominância em crianças de cor/raça parda e houve quatro óbitos. No que se refere aos municípios estudados, Arapiraca foi o local com maior número de casos em Alagoas.

Dessa forma, os resultados sinalizam para a necessidade de maior aprofundamento no estudo das variáveis que se relacionam as internações hospitalares por diarreia e gastroenterites, uma vez que a literatura aponta para associação da doença ao deficiente planejamento de saneamento básico. Portanto, é imprescindível que se conheça o perfil epidemiológico e as variáveis envolvidas no acometimento da diarreia em crianças nesta faixa etária, para que as informações obtidas subsidiem a formulação de intervenções em saúde voltadas a prevenção desse agravo.

\section{Referências}

1. Carvalho TCN, Gabbay IB, Siqueira JAM, Linhares AC, Parente AT. Conhecimento sobre gastroenterite viral pelos profissionais de saúde de um hospital materno-infantil de referência no Estado do Pará, Brasil. Rev. Pan-Amaz Saude. [Internet]. 2014. [acesso em 2020 Jan 11]; 5(3)11-18. Disponível em: scielo.iec.gov.br/pdf/rpas/v5n3/v5n3a02.pdf.

2. Farthing M, Salam MA, Lindberg G, Dite P, Khalif I, Salazar- Lindo E, et al. Acute diarrhea in adults and children: a global perspective. World Gastroenterology Organization (WGO). J Clin Gastro enterol. [Internet]. 2012. [acesso em 2020 Jun 10]; 47 (1)12-20. Disponível em: https://www.worldgastroenterology.org/guidelines/globalguidelines/acute-diarrhea/acute-diarrhea-english.

3. Rodrigues JRP, Strinta L, Silvestre GCSB, Junior JCO, Barros LC. Diarreia em crianças menores de cinco anos em uma unidade de saúde da família. Rev. Enferm UFSM [internet]. 2014. [acesso em 2020 Jan 11]; 4(3) 594-601. Disponível em: https://periodicos.ufsm.br/reufsm/article/view/13490.

4. Oliveira BSB, Oliveira RKL, Bezerra JC, Melo FMS, Monteiro FPM, Joventino ES. Condições sociais e condutas maternas na prevenção e manejo da diarreia infantil. Cogitare Enferm. [Internet]. 2017. [acesso em 2020 Jan 11]; 22(4) 1-9. Disponível em: https://revistas.ufpr.br/cogitare/article/view/50294.

5. Alencar IZ, Viana VR, Malheiro DR, Santos FAV. Ausência de saneamento básico e sua relação com a diarreia em crianças no nordeste brasileiro, nos anos de 2007 a 
2019: uma revisão de literatura. Rev. Estação Científica [Internet]. 2019 [acesso em 2020 Jan 31]; (22). Disponível em: https://portal.estacio.br/media/4681216/ausênciade-saneamento-básico-e-sua-relação-com-a-diarreia-em-crianças.pdf.

6. Brasil. Instituto Brasileiro de Geografia e Estatística (IBGE). Pesquisa de informações básicas municipais. Perfil dos Municípios Brasileiros. Saneamento Básico: aspectos gerais da política de saneamento básico. [Internet]. 2017. [acesso em 2020 Jan 03]; Disponível em: https://biblioteca.ibge.gov.br/visualizacao/livros/liv101610.pdf.

7. Brasil. Ministério da Saúde. Departamento de Informática do SUS (DATASUS). [Internet]. 2020 [acesso em 2020 Jan 03]; Disponível em: http://www2.datasus.gov.br/DATASUS/index.php?area=02.

8. Bonita R.; Beaglehole R.; Kjellstrom T. Epidemiologia Básica. $2^{\mathrm{a} e d . ~ S a ̃ o ~ P a u l o, ~ S a n t o s, ~}$ 2010 .

9. Prezotto KH, Chaves MMN, Mathias TAF. Hospitalizações sensíveis à atenção primária em crianças, segundo grupos etários e regionais de saúde. Rev. esc. enferm. USP [Internet]. 2015 [acesso em 2020 Ago 25];49(1)44-53. Disponível em: http://www.scielo.br/scielo.php?script=sci_arttext\&pid=S0080$62342015000100044 \& \operatorname{lng}=$ en.

10. Oliveira TCR, Latorre MRDO. Tendências da internação e da mortalidade infantil por diarréia: Brasil, 1995 a 2005. Rev Saúde Pública [Internet]. 2010. [acesso em 2020 Jan 20]; 44(1) 102-111. Disponível em: http://www.scielo.br/pdf/rsp/v44n1/11.pdf.

11. Oliveira RKL, Oliveira BSB, Bezerra JC, Silva MJN, Melo FMS, Joventino ES. Influência de condições socioeconômicas e conhecimentos maternos na autoeficácia para prevenção da diarreia infantil. Esc Anna Nery [Internet]. 2017. [acesso em 2020 Jan 11]; 21(4) 1-9. Disponível em: http://www.scielo.br/pdf/ean/v21n4/pt_1414-8145ean-2177-9465-EAN-2016-0361.pdf.

12. Rocha SRF. Estudo epidemiológico e etiológico de crianças com diarreia aguda por norovírus e outros agentes em unidade de emergência pediátrica, Salvador Bahia. Bahia. Monografia [Graduação em Medicina] - Faculdade de Medicina da Bahia; 2012.

13. César, MLVS. Doença diarreica aguda: aspectos epidemiológicos e vigilância no município de Avaré, interior do estado de São Paulo. São Paulo. Dissertação [mestrado em Saúde Pública] - Universidade de São Paulo; 2006.

14. Brasil. Instituto Brasileiro de Geografia e Estatística (IBGE). Portal Cidades. Brasil em síntese. [Internet]. 2019. [acesso em 2020 Jan 03]; Disponível em: https://cidades.ibge.gov.br/brasil/al/arapiraca/panorama.

15. Feitosa A, Ferreira AS, Correia JA, Lopes, JLS. O comprometimento das águas do riacho Piauí em Arapiraca/AL: causas e consequências. Braz. J. of Develop [Internet]. 2020. [acesso em 2020 Jun 03]; 6(1)2227-2242. Disponível em: brjd.com.br/index.php/BRJD/article/view/6133/5455.

16. Rufino R, Gracie R, Sena A, Freitas CM, Barcellos C. Surtos de diarreia na região Nordeste do Brasil em 2013, segundo a mídia e sistemas de informação de saúde Vigilância de situações climáticas de risco e emergências em saúde. Ciência\& Saúde 
Coletiva [Internet]. 2016. [acesso em 2020 Jan 31]; 21(3) 777-788. Disponível em: http://www.scielo.br/pdf/csc/v21n3/1413-8123-csc-21-03-0777.pdf.

17. Alagoas. Secretaria de Estado da Saúde. Superintendência de Planejamento e Participação Social. Plano Diretor de Regionalização da Saúde de Alagoas [Internet]. 2011 [acesso em 2020 Jan 20]; Disponível em: https:/www.saude.al.gov.br/wpcontent/uploads/2020/06/pdr_2011.pdf.

18. Meneguessi GM, Mossri RM, Segatto TCV, Reis PO. Morbimortalidade por doenças diarreicas agudas em crianças menores de 10 anos no Distrito Federal, Brasil, 2003 a 2012. Rev. Epidemiol. Serv. Saúde. [Internet]. 2015. [acesso em 2021 Mar 23]; 24(3):721-730. Disponível em: http://scielo.iec.gov.br/scielo.php?script=sci_arttext\&pid=S167949742015000400014.

19. Araújo MMP, silva CG. A importância do sistema de informação de agravos de notificação - SINAN para a vigilância epidemiológica do Piauí. Rev. Interdisciplinar Ciências e Saúde [Internet]. 2015 [acesso em 2020 Jan 20]; 2(3). Disponível em: https://revistas.ufpi.br/index.php/rics/article/view/2046.

20. Fabri ACOC, Alves MS, Faquim LJ, Oliveira MLL, Freire PV, Lopes FN. Cuidar em Enfermagem: Saberes de enfermeiros da Atenção Primária a Saúde. Rev. Enferm. UFPE online. [Internet]. 2013. [acesso em 2020 Jun 10]; 7(2)474-480. Disponível em: https://webcache.googleusercontent.com/search?q=cache:4ymp5WBr5mkJ:https://peri odicos.ufpe.br/revistas/revistaenfermagem/article/download/10257/10877+\&amp;cd= 1\&amp;hl=pt-BR\&amp;ct=clnk\&amp;gl=br.

\section{Como citar este artigo:}

Costa CMO, Santos DF, Bulhões TMP, Oliveira JO, Gusmão BR, Oliveira JBV, Nascimento RZ, Costa CRB. Perfil epidemiológico da diarreia em crianças de 1 a 4 anos no estado de Alagoas. Rev. Aten. Saúde. 2021; 19(68): 89-97. 